Journal of The Korean Astronomical Society

38: $289 \sim 290,2005$

\title{
SN 1604 IN CHINA
}

\author{
Z.R. WANG ${ }^{1}, Y$ Y. ZHAO ${ }^{2}, M \cdot \mathrm{LI}^{1}$, AND Q.L. ZHOU \\ ${ }^{1}$ Department of Astronomy, Nanjing University, Nanjing, 210093 \\ ${ }^{2}$ Institute Of Chinese Classics, Nanjing University Nanjing, 210093 \\ (Received February 1, 2005; Accepted March 15, 2005)
}

\begin{abstract}
The 6th East Asian Meeting of Astronomy was held just at the time of 400 years after the discovery of SN (supernova) 1604 and its pre-maximum observation by the astronomers both from the East and the West in the 17th century. It has a special meaning and is interesting to look back on the historical observation of SN 1604. In this paper, we only limit to concern the Chinese observation on SN 1604.
\end{abstract}

Key words : supernova - historical supernova:SN 1604

SN 1604 appeared in Ming Dynasty, during Wanli 32nd to 33rd year of Emperor Shengzong reign period. The first observation of it was on Oct. 10th 1604 and was recorded in Ming Shenzong Shilu Vol. 400 (see Fig.1) and the last observation was on Oct. 7th, 1605 recorded in vol. 412 (Fig.3). An observation during the middle period (Feb. 3rd, 1605) was recorded in vol.404 (Fig.2). It is obvious that the Chinese astronomers at that time kept observing SN 1604 nearly one year. The summary for the whole year's observation of SN 1604 was originally recorded in Ming Shenzong Shilu vol. 412 (Fig.3), also in Mingshi vol. 27 (Fig4). From the above records, it can be seen that SN 1604 was a guest star fixed in Lunar Mansion of Wei with an explosion phenomenon just like a pellet bomb and was visible for about one year. The visual direction and position of SN 1604 are emphasized in the records, whenever it appeared, disappeared and reappeared, accompanied with the rotation of the sky. It seems that the Chinese astronomers at that time had understood the movement of the celestial sphere as a reflection of the spinning of the earth and its rotation around the sun.

\section{ACKNOWLEDGEMENTS}

Thanks for the organizers of the meeting for their invitation and partial financial support. The research is supported by NNSF in China.

\section{REFERENCES}

Ho, P. Y. \& Chiu, L.Y., 1986, Astronomical Records in the Ming Shilu (Hong Kong Univ. press)

Stephenson,F.R. \& Green, D.A. 2002, Historical Supernova and their Remnants (Oxford)

Xi, Z. Z. \& Bo, S. R. 1965 Acta Astronomica in Sinica, 13,

Proceedings of the 6th East Asian Meeting of Astronomy, held at Seoul National University, Korea, from October 18-22, 2004.

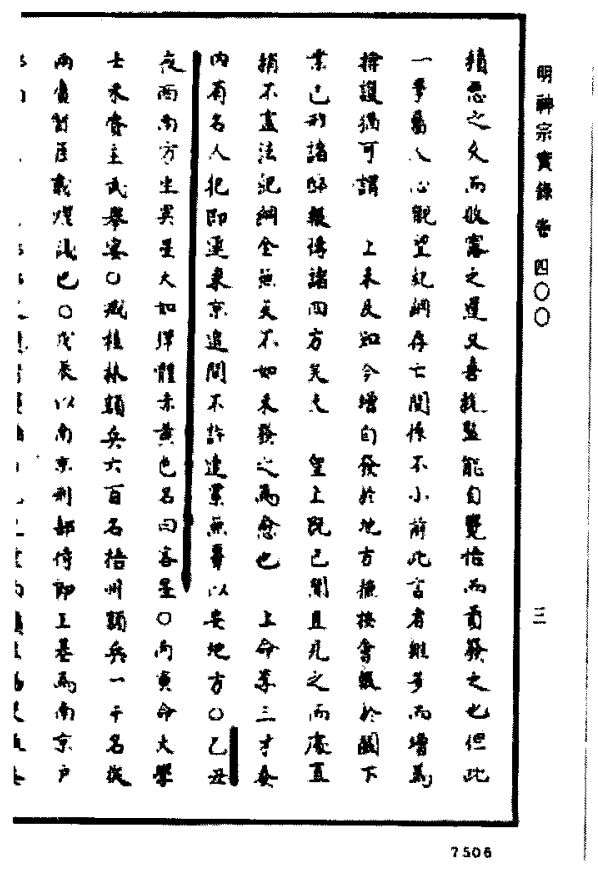

Fig. 1.- The record of SN 1604 for its first appearance in Ming Shengzong Shilu On Oct. 10th, 1604 (day yichou, 9th lunar month in 32nd year of Wanli): "At night, a strange star as large as a pellet bomb appeared at the SW direction, with orange color and was called a 'Guest star'".

Wang C.W. 1940, Ming Shengzong Shilu (Viritable records of the reign period of the King Shengzong), Vol 400-412

Wang, Z.R., Zhao, Y, Li, M. \& Zhou, Q.L., 2004, in 16042004 Supernovae as Cosmological Lighthouses (in press)

Zhang, T.Y., 1739, Ming Shi (History Of Ming Dynasty), Vol 27 


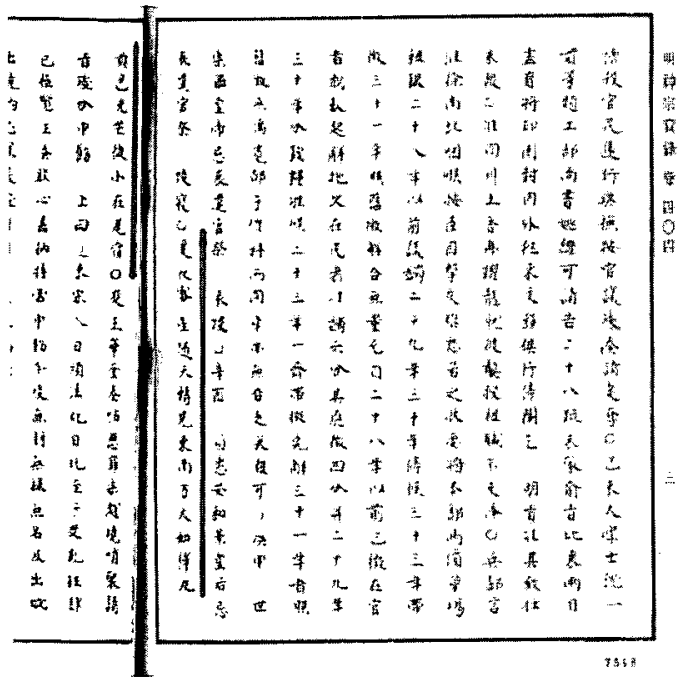

Fig. 2.- The record of SN 1604 on Feb. 3rd, 1605 (day Xinyou, 12th lunar month in 32 nd year of Wanli): "Tonight, the guest star was seen at SE direction rotated with the sky..., as large as a pellet bomb with yellow color and became less brightness in the Lunar Mansion of Wei."

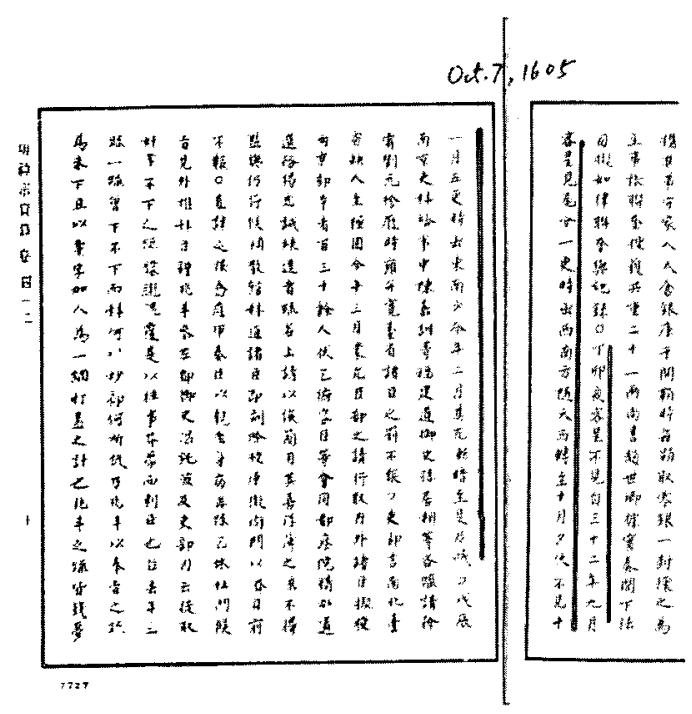

Fig. 3.- The record of SN 1604 on Oct. 7th, 1605 (day dingmao, 8th lunar month in 33rd year of Wanli) and the summary of its nearly whole year's observation: "Tonight, the guest star was out of sight. It was seen in the Lunar Mansion of Wei since Oct. 10th, 1604, usually appeared at the SW direction at $7-9 \mathrm{pm}$, rotated westward with the sky until 10th lunar month it sank and was not seen in the evening. In the 11 th lunar month, it usually appeared at $3-5 \mathrm{am}$ at the SE direction. During the 2nd lunar month of the current year it became faint and now has been fully extinguished."

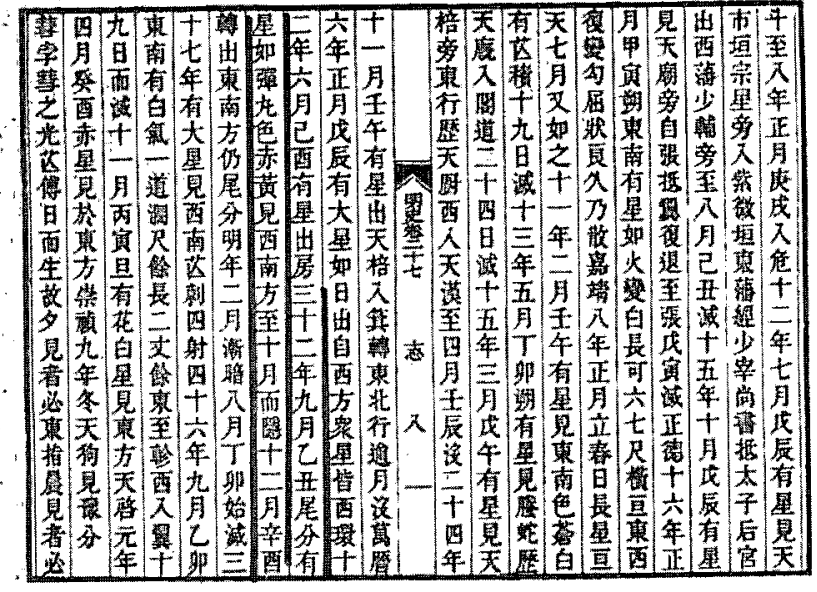

Fig. 4.- The record of SN 1604 in Ming Shi on summary of its nearly whole year's observation "On Oct. 10th, 1604, there was a star as a pellet Bomb with yellow color appeared in the SW direction in the lunar Mansion of Wei until the 10th lunar month it was hidden from view. On Feb. 3rd, 1605 it turned round to be in SE direction still in Wei. It gradually became dim and finally extinguished on Oct. 7th, 1605." 